\title{
USING THE MANDELBROT SET TO GENERATE PRIMARY POPULATIONS IN THE GENETIC ALGORITHM AND REVERSE ORDER IN ORDER TO ENCRYPT THE IMAGE
}

\author{
Elaheh Aghamohammadi $^{1 *}$, Zeinab Amani $^{1 *}$, Maryam Rastgarpour $^{2}$ \\ ${ }^{1}$ Ph.D. student Artificial Intelligence Computer Engineer, \\ Islamic Azad University E-Campus, Tehran, Iran \\ ${ }^{2}$ Computer Engineering, Faculty Of Engineering, \\ Islamic Azad University, Saveh Branch, Saveh, Iran
}

\begin{abstract}
Nowadays, finding a way to secure media is common with the growth of digital media. An effective method for the secure transmission of images can be found in the field of visual cryptography. There is a growing interest in the use of visual cryptography in security application. Since this method is used for secure transmission of images, many of the methods are developed based on the original algorithm proposed by Naor and Shamir in 1994. In this paper, a new hybrid model is used in cryptography of images which is composed of Mandelbrot algorithm and genetic algorithm. In the early stages of proposal, a number of encrypted images are made by using the Mandelbrot algorithm and the original picture and in the next stage, these encrypted images are used as the initial population for the genetic algorithm. At each stage of the genetic algorithm, the answer of previous iterations is optimized to get the best encoding image. Also, in the proposed method, we can achieve the decoded image by a reverse operation from the genetic algorithm. The best encrypted image is an image with high entropy and low correlation coefficient. According to the entropy and correlation coefficient of the proposed method compared with existing methods, it is observed that our method gets better results in both of them.
\end{abstract}

\section{KEYWORDS}

visual cryptography, genetic algorithm, Mandelbrot function, fractal, reversible genetic algorithm.

\section{INTRODUCTION}

With the rise of digital media, the need for methods to maintain such data seem necessary. Digital media sources link to the rich source of data which are offered by the internet and the range of the data are increasing day by day. These data can be simple text documents, images of people and so on. Internet provides an easy access to this required knowledge.

Dhinaharan Nagamalai et al. (Eds) : AIS, CSIT, IPPR, IPDCA - 2017

pp. 163-182, 2017. (C) CS \& IT-CSCP 2017

DOI : $10.5121 /$ csit.2017.71014 
The field of visual cryptography is developed during the past few years. The main method was initially proposed by Naor and Shamir for binary images. This method offers a secure system in which secret messages are parts that are separately similar to random noise, but when they are properly placed on each other, their messages can be decrypted only by using the human visual system. While this method provides security for text and binary images, growth of digital media requires the development of these techniques to provide security for color and greyscale images. Through the development of the original method, visual cryptography provides a secure method to store and transfer text, binary images, gray and color images. Since the original method was developed in 1994, many changes and improvements were added to the available collection of visual encryption techniques. Many digital services require reliable security for the transfer and storage of digital images. Due to the rapid growth of the Internet in today's digital world, security of image has attracted a lot of attention. Prevalence of multimedia technologies in our society is caused digital images play a substantial role compared to the old texts which calls for serious protection of users' privacy is for all applications. Digital images' encryption techniques are very important that should be protected against unauthorized access attacks.

Digital images are exchanged on various types of networks that often a large part of these data is confidential or private. Encryption is a preferred technique to protect the transmitted data. There are various encryption systems to encrypt and decrypt data image. Today, images can be regarded as one of the usable forms of information. Image Encryption has various applications in different fields such as internet communications, multimedia systems, medical imaging, telemedicine and military communications and therefore, providing effective and secure protection for image files is one of the main concerns.

In proposed method, at first, the input images are combined with Mandelbrot fractal image then in terms of the size of the images, a series of random numbers is generated and in the next stage, pixel is selected with the use of these numbers in both of the rows and combined dual-point operation is performed on them. And finally, by using random numbers generated in the second step, the row of image pixels of previous step is dislocated that this act is similar to the mutation function in the genetic algorithm and in this way the encrypted image is obtained and if input images to be colorful, these acts are done on all three color components. In the decode step, at first the mutation function conducted at the encryption step is carried out inversely then, the two point combined action is also performed inversely. Finally, in order to achieve the original image, the Mandelbrot fractal image is subtracted from the generated image.

\section{ENCRYPT IMAGES}

The need for image encryption to securely transfer images across communication channels, such as Internet and wireless communications networks, is increasing, and due to the large amount of video and image data of traditional cryptographic algorithms, they do not have the required efficiency in this area. In this dissertation, a new method is proposed based on the integration of the Mandelbrot fractal image and genetic operators, which has the ability to encode images with a minimum correlation coefficient and maximum entropy. 


\section{Frectal}

In mathematics, the object itself is exactly or nearly identical to that of itself. For example, a shape has the form of someone like its own parts or parts.

Many real-world objects, such as coastlines, are statistically similar, with some of them showing the same statistical characteristics on many scales. Stable on a scale or scale is a precise form of self-similarity. So that at each magnification there is a smaller piece of object that is geometrically similar or similar to the general one. Instead, one side of the snowflake, which is both symmetrical and stable, can grow steadily without change in shape.

A compact space with its topology $\mathrm{X}$ is the same if there is a set of bounded Ss that shows a disjoint cluster set. For If, we call ourselves the same as $\mathrm{X}$ if and only if the subgroup of $\mathrm{Y}$ is such that the equation is reserved for the above equation. In this case we have:

A self-constructed structure, such as a homogeneous one, may be a repeating function, which is the result of a repeating function system. A combination of functions of a monoidal algebraic structure creates. When the $\mathrm{S}$ set has only two factors, monoids are known as dynamic monoids. A dynamic monoid can be represented as infinite binary tree. In general, if the set $\mathrm{p}, \mathrm{S}$ is a factor, the monoid may be represented as a p-adic tree. The Mandelbrot set is similar to its chihuahua points.

Self-similarity has important implications for the design of computer networks, and the typical network of traffic has its own characteristics. For example, in remote traffic engineering, packet switched data traffic patterns seem to be statistically similar. This means that the use of simple Poisson distribution models is inaccurate, and that networks that are designed to operate unexpectedly, regardless of self-similarity, are to some extent unexpected. Similarly, stock market volatility is defined as a self-similarity drama. For example, when they move from a special anchor conversion to a more detailed display, they appear as if they were. Many of their objects are like natural herbs. Real ferns are much closer to real self-similarity. Other plants, like flowers, are largely the same

\section{Mandelbrot fractal model}

Mandelbrot, when studying a study of the length of British coasts, concluded that when measured on a large scale this length is greater than that at a smaller scale. This disorder caused the creation of a mathematical branch of the disorder theory called fractal. This term was first introduced in 1975 by the Polish mathematician, Bennett Mendel Brut. The term fractal is derived from the Latin Latin fractus or fractal meaning a broken or broken broken stone. The Dictionary of Persian and Farsi has approved the word bhakal for fractals. Fractals are the forms that, unlike the Euclidean geometric forms, are not at all regular. These forms are, firstly, all irregular and, secondly, their degree of disorder is the same at all scales. In his explanation of his theory, Mandelbrot emphasized the choice of the phrase fractal on one of the main characteristics of this geometric form, which is due to the nature of the piece, fragmentation. In his opinion, the universe and all natural phenomena are somewhat fractal. He has stated that the clouds are not like Korea, the mountains are not like cones, the shores of the sea are not circular, the skin of the tree is not smooth, and the lightning does not move in a straight line. Observing the nature of the nature, it is clear that Euclidean geometry can not explain the complex and apparently 
disorganized forms. Euclidean geometry (the full sphere of spheres, pyramids, cubes and cylinders) is not the best way to show natural elements. The clouds, the mountains, the coastline and the trunk of trees are all contradictory with Euclidean currents, and not smooth, but rugged, and this disorder brings in small scales, which is one of the most important features of fractals. This means that fractal geometry, in contrast to Euclidean geometry, is a better way of explaining and creating phenomena like nature. The language to which this geometry is expressed is called an algorithm that can be translated into simpler formulas and rules with compound objects. Fractals are elements whose spatial form is not smooth. Therefore, they are also called "irregular", and this irregularity is repeated geometrically and in a variety of scales within the pyramid. Every natural thing around us is essentially a fractal. Because straight lines and planes exist only in the ideal mathematical world. Along with this theory, any system that can be geometrically imagined and analyzed can be a fractal. The world in its general form of physical (material) is chaotic, irregular, irregular, but behind this initial mentality there is a coherent and conservative law that is based on order and has a clear combination. The best way to define a fractal is to pay attention to its attributes and symptoms. An "irregular" fractal means that there is no flat part in it. The fractal is "self-similar", which means that the "components" are similar to the whole. The fractal body is seen from far and near the same. In other words, it is similar. When we approach a fractal object, small pieces of it from the round are thought to be massive grains, they are physically depicted in a manner more or less like the image seen from the far side. In nature, there are many examples of fractals. Trees, clouds, mountains, rivers, the edges of the beaches, and bullets are all fractal objects. A small part of a tree whose branch is similar to the whole tree. This example can be used for clouds, bullets, thunderbolts and other fractal objects. Many artificial human hand elements are also fractal. Celican chips, the stock market volatility curve, the growth of cities, and finally the Sarpinsky triangle. The Serpinsky Triangle is an equilateral triangle, with the points in the middle of its side connected to each other. If this action continues within the triangles of the new trivial to infinite, triangles are always obtained that are similar to the first triangle. In mathematical science, fractal is a complex engineering complex with similar details in its structure on any scale. The degree of disorder is far from one and the same .

Mandelbrot, when studying a study of the length of British coasts, concluded that when measured on a large scale this length is greater than that at a smaller scale. This disorder caused the creation of a mathematical branch of the disorder theory called fractal. This term was first introduced in 1975 by the Polish mathematician, Bennett Mendel Brut. The term fractal is derived from the Latin Latin fractus or fractal meaning a broken or broken broken stone. The Dictionary of Persian and Farsi has approved the word bhakal for fractals. Fractals are the forms that, unlike the Euclidean geometric forms, are not at all regular. These forms are, firstly, all irregular and, secondly, their degree of disorder is the same at all scales. In his explanation of his theory, Mandelbrot emphasized the choice of the phrase fractal on one of the main characteristics of this geometric form, which is due to the nature of the piece, fragmentation. In his opinion, the universe and all natural phenomena are somewhat fractal. He has stated that the clouds are not like Korea, the mountains are not like cones, the shores of the sea are not circular, the skin of the tree is not smooth, and the lightning does not move in a straight line. Observing the nature of the nature, it is clear that Euclidean geometry can not explain the complex and apparently disorganized forms. Euclidean geometry (the full sphere of spheres, pyramids, cubes and cylinders) is not the best way to show natural elements. The clouds, the mountains, the coastline and the trunk of trees are all contradictory with Euclidean currents, and not smooth, but rugged, and this disorder brings in small scales, which is one of the most important features of fractals. This means that fractal geometry, in contrast to Euclidean geometry, is a better way of explaining 
and creating phenomena like nature. The language to which this geometry is expressed is called an algorithm that can be translated into simpler formulas and rules with compound objects. Fractals are elements whose spatial form is not smooth. Therefore, they are also called "irregular", and this irregularity is repeated geometrically and in a variety of scales within the pyramid. Every natural thing around us is essentially a fractal. Because straight lines and planes exist only in the ideal mathematical world. Along with this theory, any system that can be geometrically imagined and analyzed can be a fractal. The world in its general form of physical (material) is chaotic, irregular, irregular, but behind this initial mentality there is a coherent and conservative law that is based on order and has a clear combination. The best way to define a fractal is to pay attention to its attributes and symptoms. An "irregular" fractal means that there is no flat part in it. The fractal is "self-similar", which means that the "components" are similar to the whole. The fractal body is seen from far and near the same. In other words, it is similar. When we approach a fractal object, small pieces of it from the round are thought to be massive grains, they are physically depicted in a manner more or less like the image seen from the far side. In nature, there are many examples of fractals. Trees, clouds, mountains, rivers, the edges of the beaches, and bullets are all fractal objects. A small part of a tree whose branch is similar to the whole tree. This example can be used for clouds, bullets, thunderbolts and other fractal objects. Many artificial human hand elements are also fractal. Celican chips, the stock market volatility curve, the growth of cities, and finally the Sarpinsky triangle. The Serpinsky Triangle is an equilateral triangle, with the points in the middle of its side connected to each other. If this action continues within the triangles of the new trivial to infinite, triangles are always obtained that are similar to the first triangle. In mathematical science, fractal is a complex engineering complex with similar details in its structure on any scale. The degree of disorder is far from one and the same.

\section{IMAGE DECODING}

In this section, all the processes and processes in the encryption section are reversed. First, the encoded image is loaded and the Fibonacci random string is created using the golden code, and then the image of the fractal is created. In the next step, based on the generated string, the mutation operation is reversed and the encryption section is applied to the encrypted image. Then the intersection operation is inversely applied to the pixel rows of the encrypted image. And these apply to all the pixels in the images. So, in order to achieve the original image, we need to subtract the image of Mendel Bert's fractal image from the encoded image, which is done by subtracting the pixel into the pixel and capturing the original image.

\section{CRYPTOGRAPHY}

Cryptography is the science of codes and ciphers and an ancient art and it is used for centuries to protect the messages that were exchanged between the commanders, spies, lovers and others to make their messages confidential. When data security is discussed, it is necessary to prove the identity of the sender and receiver of the message and also, to be sure not to change the content of the message. The three issues of confidentiality, authentication and integrity are at the heart of modern data communications security and can use encryption (8). This issue should be ensured that a message can only be read by those for whom the message was sent, and others are not allowed. Cryptography is the provider of this issue. 
Cryptography is the art of writing in an encrypted format so that no one except the intended recipient cannot read the message content. Cryptography has two main components including algorithm and key. The algorithm is a converter with mathematical formula. The key is a string of binary digits (one and zero) which in itself is meaningless (7). Modern cryptography assumes that algorithm is known or can be discovered. It is the key that should be kept secret or varies at each stage of implementation. Decryption may use the same pair of algorithm and key or different pair $(1,2)$.

\section{Areas of Cryptography}

Issues related to the field of cryptography can be proposed and considered at different levels (7). At the first level, a number of the paradigm such as symmetric cryptography, asymmetric cryptography and mixture exist and at the second level, a number of algorithms such as RSA and DES exist and protocols and standards are established at the third level. At the fourth level, applications or other protocols are created on these protocols. Maybe algorithm to be good, the protocol to be well-defined and established, but at the level of implementation of the application not to be good. Therefore, every level requires a degree of quality, performance, and confidence and creating influence on the systems may naturally return to each of these levels (2).

\section{The terms used in Cryptography}

Cryptology: The science of the study of cryptography and decryption

Password device: a system that is created to encrypt and decrypt data.

Cryptography: It is the art and science of mathematical techniques related to the concepts of data security like confidentiality, data integrity, authentication, non-repudiation.

Decryption: The study of methods that are used to break the encryption techniques Cryptographer: A person who is studying the systems and versions of encryption.

Decrytor: A person who is malicious at decoding and analysis of codes.

Cryptography: The encryption process of messages in a way that it's content to be hidden from foreigners.

Decryption: The process of recovering plaintext from the ciphertext.

Symmetric encryption algorithm: symmetric encryption algorithms or private key encryption use a key to encrypt and decrypt of data.

Asymmetric cryptography algorithm: Asymmetric Cryptography algorithm or public key cryptography algorithm uses different keys to encrypt and decrypt of data and decryption key cannot be derived from the encryption key $(1,3)$.

DES, Triple-DES, SHA-1, RSA are the public key algorithm and RSA is the most famous public key algorithm that is used for encryption and digital signature. RSA calculations are done with integers $\mathrm{n}=\mathrm{p} * \mathrm{q}$ for large prime numbers $\mathrm{p}$ and $\mathrm{q}$. To encrypt the message of $\mathrm{m}$, it reaches to the view of a public small view of e. 
To decrypt, the receiver of cipher text of $C=\operatorname{me}(\bmod n)$, calculates reverse of $d=e-1(\bmod (p-1)$ $*(\mathrm{q}-1))$ and obtains the amount of $\mathrm{Cd}=\mathrm{me}^{*} \mathrm{~d}$. The private key includes $\mathrm{m}(\bmod \mathrm{n}) \mathrm{e}, \mathrm{q}, \mathrm{n}, \mathrm{p}, \mathrm{d}$. The public key only includes e, $\mathrm{n}(1,13)$.

\section{Cryptographic Methods}

Symmetric method: In this method both sender and receiver of information have a common key for encryption and decryption. In this state, the encryption and decryption of information are two reverse process. Key transition between each other by intranet or physically is somewhat safe. But its transmission by internet is not correct (4). In such systems, the keys for encryption and decryption are same or have very simple relationship with each other. Symmetric encryption is used to encrypt large amounts of data. When it is used with a digital certificate, the confidentiality of information is protected. When electronic signature is used, the message integrity is guaranteed (2).

Advantages: High speed during the encryption, key generated randomly and fast.

Disadvantages: a plurality of keys for the members of each connection, key distribution among communicating parties.

Asymmetric method: This method was created to solve the problem of key transfer in symmetric method. In this method, instead of a shared key a pair of public and private key is used. In this method, the public key is used for encryption of information. The one who intends to transfer information in an encrypted form, encodes information and sends to the person who is the owner of the key pair. The owner of key maintains the private key for himself in a confidential form. In this method, the encryption and decryption keys are distinct (2).

Advantages: No need to distribute and send key.

Disadvantages: low speed in high volume of data, the complexity of key generation.

\section{The suggested process for image encryption in this method}

The need for image encryption to securely transmit images over communication channels such as internet networks and wireless communications networks is increasing and due to the high volume of image and video data the traditional encryption algorithms do not enjoy the necessary efficiency in this regard (53).

In this study, a new method is proposed based on a combination of Mandelbrot fractal image and genetic operators with the ability to encrypt images with minimal correlation coefficient and maximum entropy. In Figure 1, the overall chart of image encryption stages of the proposed method is displayed. Then, the stages of image encryption system are completely explained.

\section{Combining the original image with the image of Mandelbrot}

At this stage, the input images with Mandelbrot fractal image that have the same size are gathered pixel by pixel and the remaining pixel of original image with pixel of Mandelbrot image is calculated to number of 255 for each pixel of output image. And for color images, these actions are calculated for triple RGB colors. 


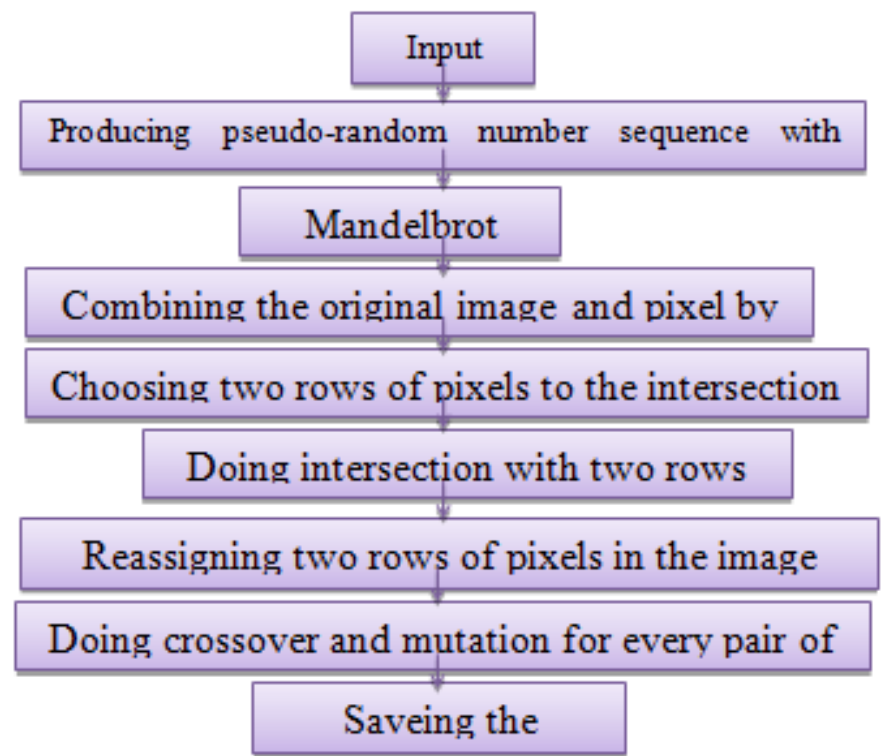

Figure 1. The overall chart of the encryption system of the proposed system

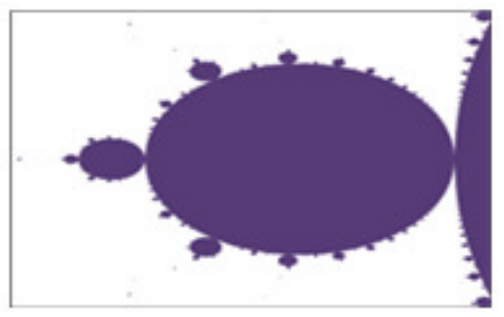

Figure 2. Self-resemblance in Mandelbrot collection.

\section{Numerical code generation for encryption and decryption}

At this stage, a series of Fibonacci random numbers are produced in relation to the size of the input images and the golden key that these numbers are used in the later stages.

\section{Fibonacci series}

In mathematics, Fibonacci series are a sequence of numbers that are defined as follows:

$$
F(n):= \begin{cases}0 & \text { if } n=0 \\ 1 & \text { if } n=1 \\ F(n-1)+F(n-2) & \text { if } n>1\end{cases}
$$

In which except the two prime numbers, the next numbers are obtained from sum of their previous two numbers. The first numbers of the series are: 1, 1, 2, 3, 5, 8, 13, 21, 34, 55, 89, 144, $233,377,610,987,1597,2584, \ldots$. These numbers are named to the name of Italian mathematician Leonardo Fibonacci.

\section{Double point crossover operator on rows of output images}

At this stage, by using the code generated in previous stage, at first, two rows of pixels as two chromosomes become ready for crossover action and to determine the crossover pixel, at first, the number of row of each chromosome is divided to 255 and is multiplied in the number of pixel of 
each row (the number of genes) and the number of pixel is achieved for double point crossover action. This is achieved for all pixel rows and for each three RGB color channels.

\section{Mutation operator on the output images}

At this stage, by using the code generated in the second stage, the row of pixels of images of previous step is dislocated and this is obtained for all row of pixels and for three RGB color channels. In Figure 3, an example of mutation operator is shown on the two rows of pixels of the image.

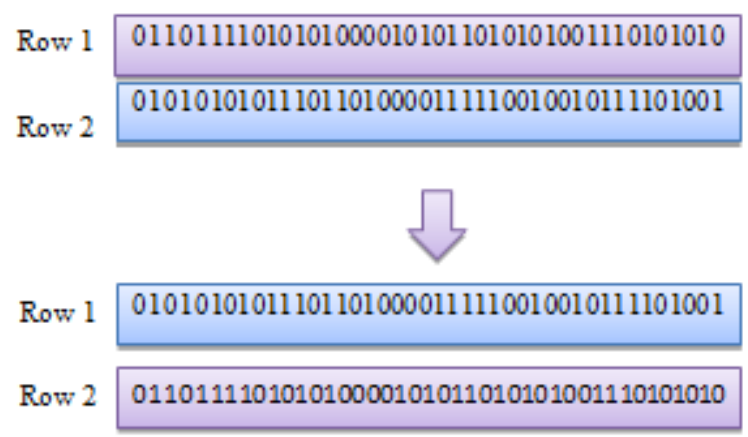

Figure 3. An example of mutation operator on two rows of pixels of the image

Finally, the encrypted image is created by using a combination of input images with Mandelbrot fractal image and operators of genetic algorithm. Figure 4 shows an example of the input image and the encrypted image
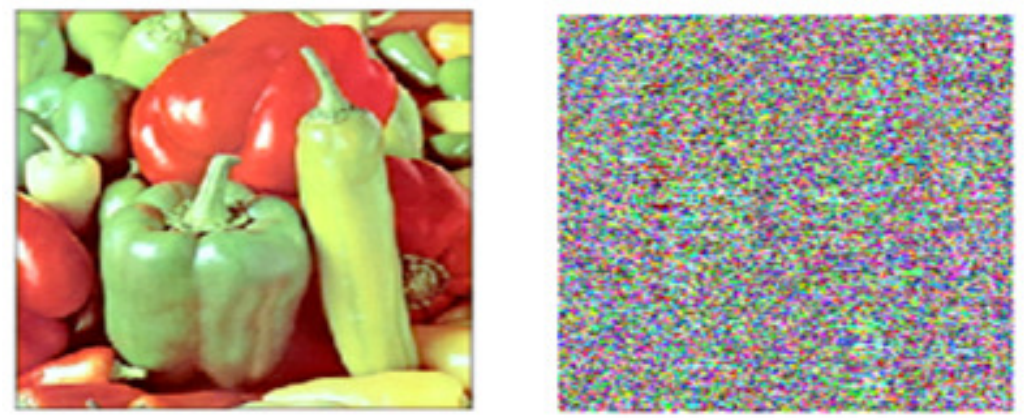

Figure 4. An example of the input image and the encrypted image

\section{Decoding image}

In this section, all the steps and processes in the encryption are performed inversely. At first, the encrypted image is loaded and the sequence of Fibonacci random numbers are created by using the golden code, then the Mandelbrot fractal image is created. In the next stage, based on the sequence of produced numbers, mutation operation is inversely applied and contrary to the encryption part on encrypted image. Then, the crossover practice is also applied in reverse form on pixel rows of encrypted image and this is applied to all the rows of pixels of the images. Finally, in order to achieve the main image the Mandelbrot fractal image should be subtracted from encrypted image and this action is done by pixel by pixel subtraction. Figure 5 shows the overall chart of steps involved in images decoding. In the following the details of each section are explained. 


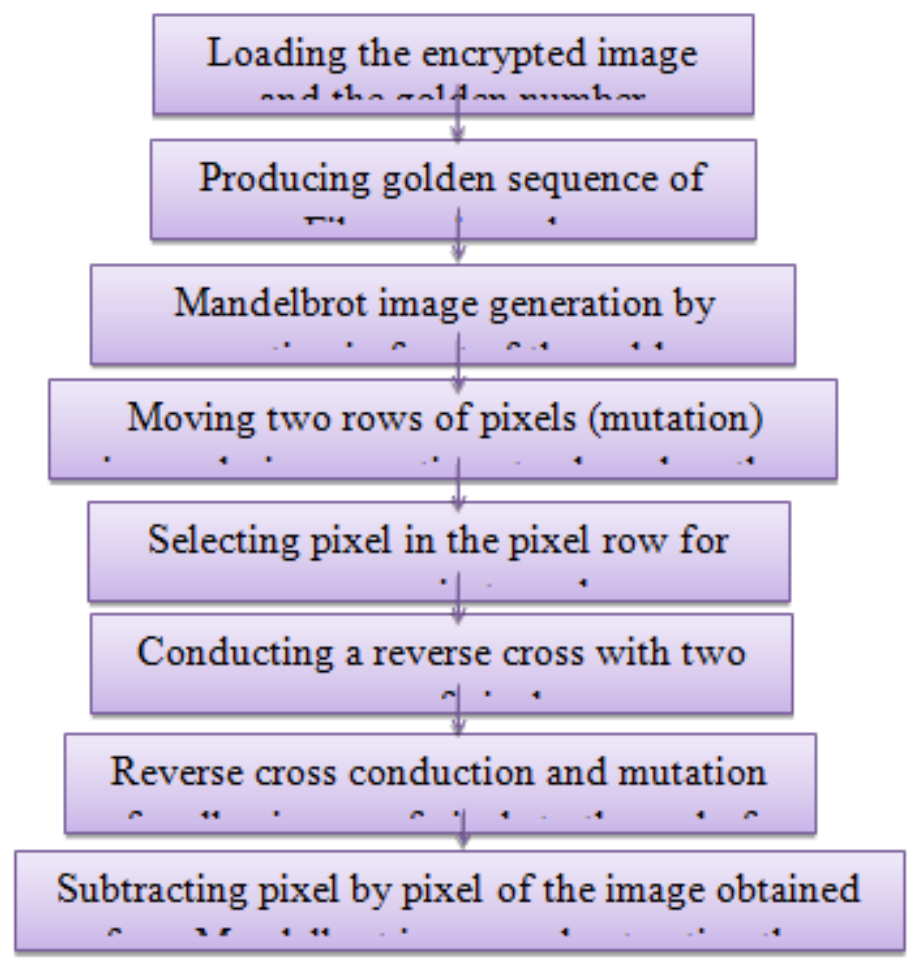

Figure 5. The overall chart of decoding system of the proposed system.

The production of the sequence of Fibonacci random numbers

At this stage, after loading the encrypted image and the golden number, such as the encryption section, the sequence of Fibonacci random numbers is produced.

\section{Reverse mutation operator on encrypted images}

At this stage, by using a series of randomly generated code, the pixels' row of encrypted images is displaced that this operation causes the row of pixels returns to its own true location. Also, this action is performed for all rows of pixels of images and for each of the three RGB color channels.

\section{Reverse double point crossover operator on rows of encrypted images}

By using randomly generated code sequence, at first, two rows of pixel as two chromosomes become ready for crossover action and in order to determine the crossover pixel, the number of row of each chromosome is initially divided to 255 and is multiplied in the number of pixel of each row (the number of genes) and the number of pixel is achieved for double point crossover action. This action is achieved for all rows of pixels and for each three RGB color channels.

\section{Discretization of the original image from the Mandelbrot image}

At this stage, the original image is subtracted from the Mandelbrot image that are at the same size and the decoded image is obtained by combining the Mandelbrot fractal image with genetic algorithm operators. Figure 6 displays an example of the encrypted image and decrypted image. 

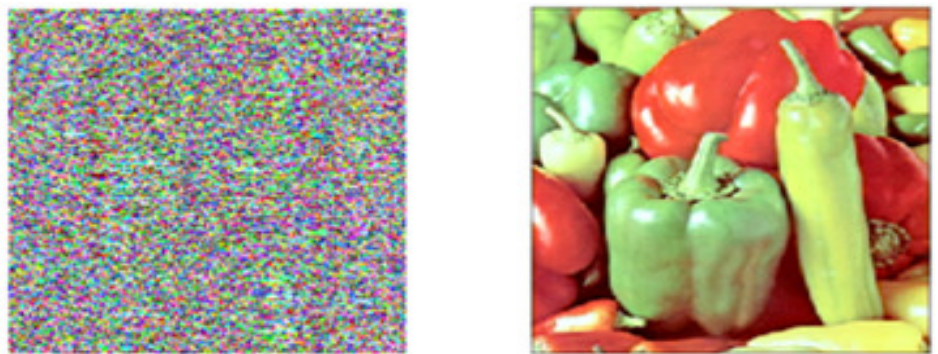

Figure 6. An example of the encrypted image and decrypted image

The performance and efficiency of proposed system are tested by using different parameters and criteria and the results obtained in each part are discussed and analyzed.

\section{Collection of images}

To evaluate and test the performance of the proposed system and also to compare with other methods the known images in this area are used. These images are being used in most of the articles and are suitable to evaluate the performance of systems. Images used in the gray area include Lena, Peppers, Baboon, House, Boat and Photographer and color images include Peppers, Baboon, Lena and Airplane. In Figure 7 the gray images used in the experiments of proposed system and in Figure 2 the color images are shown.
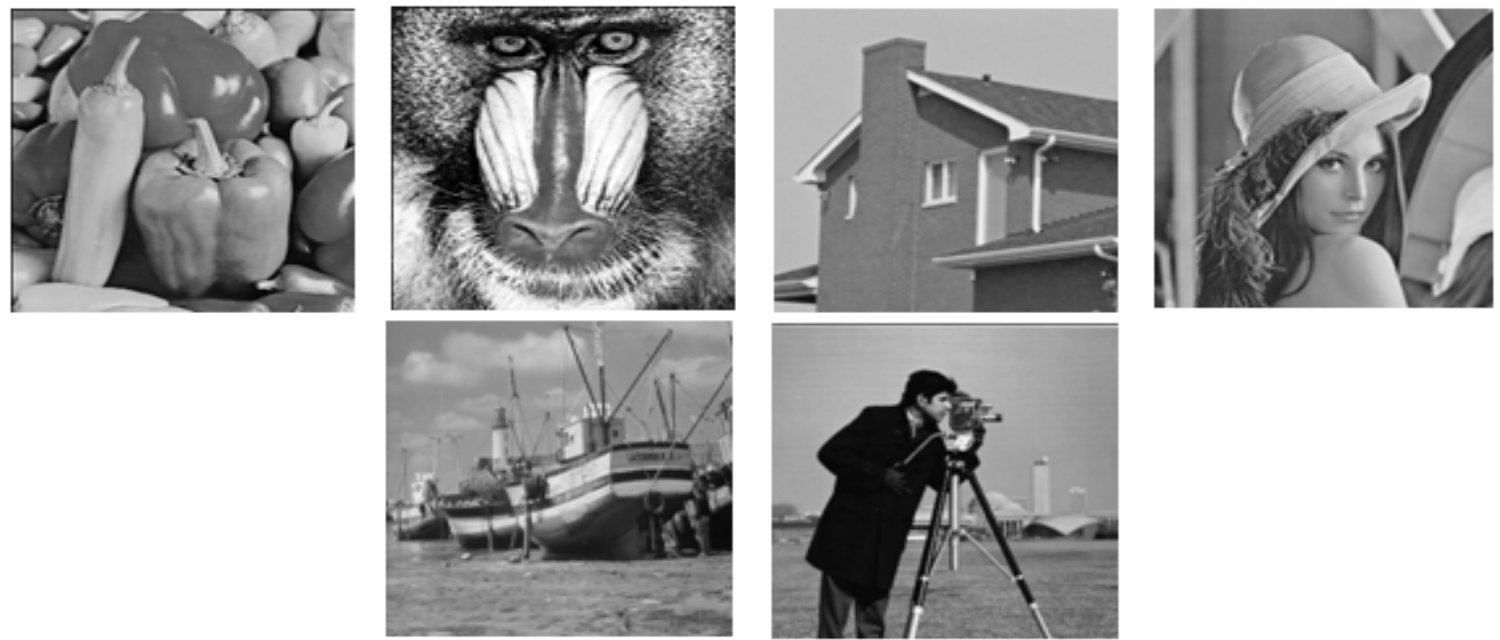

Figure 7. The gray images used in the experiments of proposed system

\section{Evaluating the proposed system}

Among the important criteria in images cryptographic operations can point to the calculation of the amount of entropy of encrypted images, correlation coefficient of the original image and encrypted image, the amount of PSNR original image and the decrypted image and also, histogram of the encrypted image. The proposed system is evaluated based on the mentioned criteria and on the gray and color images' levels. In the following the details of the results of the proposed system are presented.

\section{Entropy of the encrypted images}

Information entropy in theory of the information is in connection with the fact that a signal or a random event to what extent is random. In fact, information entropy reports the randomness rate 
as a mathematical evaluation. One of the goals of the image encryption topic is data encryption in a way that the encrypted image contains maximum entropy. In other words, the more entropy amount, the more chaos or randomness of pixels of images. This causes the information of the original image not to be recognizable from encrypted image and access to the original image by using statistical analysis to be minimized. In Table 1, the entropy rate of encoded images is shown in the sample images.

Table 1. The results of the proposed system based on the selected criteria

\begin{tabular}{|l|l|}
\hline Entropy & Input Image \\
\hline 7.8938 & Peppers \\
\hline 7.9057 & Lena \\
\hline 7.7937 & house \\
\hline 7.8940 & Baboon \\
\hline 7.8944 & Boat \\
\hline 7.9004 & Photographer \\
\hline
\end{tabular}

\section{Correlation between the original image and encrypted image}

The correlation coefficient is statistical tools to determine the relevance of a quantitative variable with another quantitative variable. The correlation coefficient is one of the criteria used to determine the correlation between two variables. It displays the intensity of correlation and this coefficient is between 1 and -1 and in the case of the lack of relationship between two variables is zero. The correlation coefficient of two images is used to investigate the relationship between the encrypted image and the original image. In our study, the aim is to reach a value close to zero and this proves the lack of connections and similarities between the two images. The correlation coefficient close to zero indicates that the encrypted images do not have the features of the original image and by using statistical analysis the original image data cannot be achieved. Table 2 displays the correlation coefficient data of the selected gray level images.

Table 2. Results of the proposed system based on selective criteria

\begin{tabular}{|l|l|}
\hline Correlation Coefficient & Input Image \\
\hline 0.0010 & Peppers \\
\hline 0.0198 & Lena \\
\hline 0.0163 & house \\
\hline 0.0111 & Baboon \\
\hline 0.0097 & Boat \\
\hline
\end{tabular}




\section{Histogram Analysis}

Histogram is a graphical representation of the number of pixels for each brightness level in the input image. For example, gray level images have 256 brightness levels, so each image pixel can have a value in the range of 0 to 255 . In the histogram, the horizontal vector is 0 to 255 and that represents the intensity of the images pixels brightness and the vertical diagram showing the frequency of the intensity of the light and the amount depends on the input images. . In the context of the encryption of images, the purpose of encoding images is to not recover any image from the original images from the encrypted image. The histogram can also display the pixel data of the image. Hence the histogram of the encoded image and the original image must be different. The more histograms the encoded images have in a more uniform state, the less information is displayed and more suitable for this field. It is also worth noting that the uniformity of the histogram diagram and the entropy of the image have a direct proportion, hence, the more entropy the image is, the more the histogram of the image becomes even more smooth. The following diagrams show histograms for main images, encrypted images and decoded images.
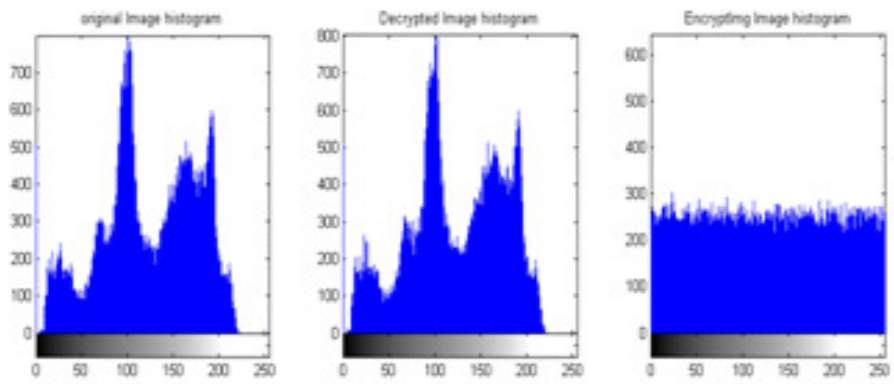

Figure (8): Histogram of the Peppers image, left histogram of the original image, decoded image and encoded image
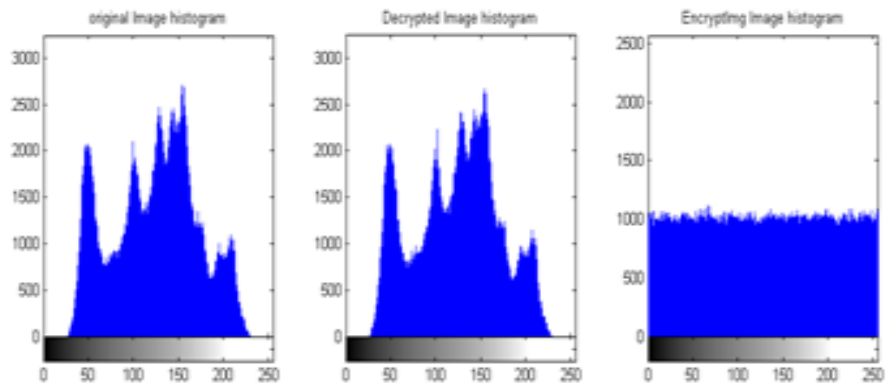

Figure (9): Histogram of the Lena image, left histogram of the original image, decoded image and encoded image.
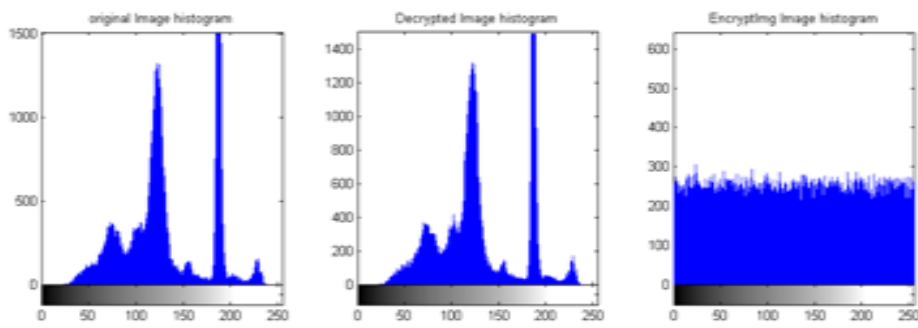

Figure (10): The histogram of the House image, from the left of the histogram of the original image, the decoded image and the encoded image 

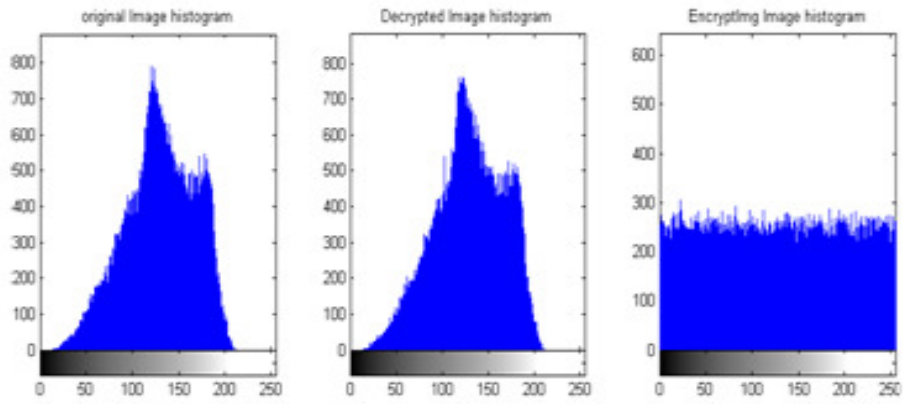

Figure (11): Histogram of the Baboon image, left histogram of the original image, decoded image and encoded image

As shown in the pictures, the histograms of the cropped images are uniform and smooth. In this case, no histogram of encrypted images can be recovered.

\section{The simulation results}

Finally, the outputs images of proposed system include encoded images and decoded images which are shown in Figure (12).
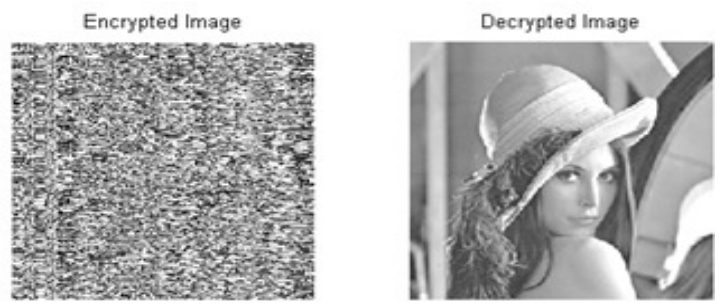

Encrypted Image

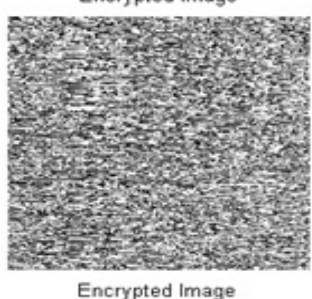

Decrypted Image

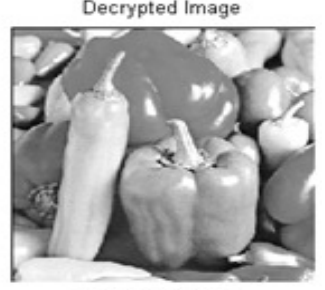

Decrypted Image
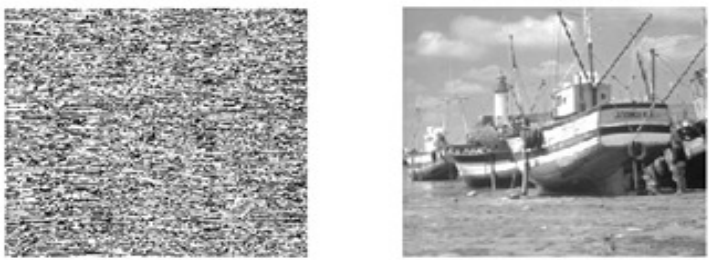

Encrypted Image

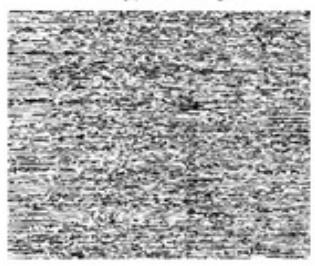

Decrypted Image

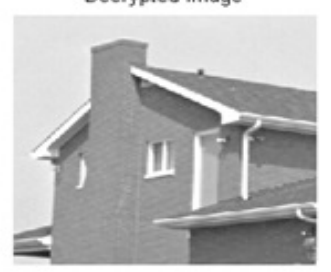

Figure 12. The outputs images of proposed system 


\section{A comparison of the proposed method and other methods}

In order to investigate the tests results of the proposed system compared to the former provided systems, the test results of previous methods are obtained based on the listed criteria. The results of the proposed system and the system (35) are displayed in the Tables 3 and 4 according to the correlation coefficient and entropy for images of Lena and Baboon.

Table 3. The results of the proposed system and system based on the selected criteria on the Lena image

\begin{tabular}{|l|l|l|}
\hline The methodused & Correlation Coefficient & Entropy \\
\hline The proposedmethod & 0.0198 & 7.9057 \\
\hline Method(56) & 0.0135 & 7.9822 \\
\hline
\end{tabular}

Table 4. The results of the proposed system and system based on the selected criteria on the Peppers image

\begin{tabular}{|c|c|c|}
\hline The method used & Correlation Coefficient & Entropy \\
\hline The proposed method & 0.0010 & 7.8938 \\
\hline Method (56) & 0.0119 & 7.9873 \\
\hline
\end{tabular}

As can be seen in Tables 5-3 to 5-4, the results of proposed system compared to the articles (56) and (57) have achieved better results in relation to the mentioned criteria.

\section{1-5-1 Results of testing the proposed system on color images}

The proposed system also has the ability to encode color images. There are three components of red, green, and blue color in the color images, each of which is a gray level image. In order to encode color images, the proposed algorithm applies to all three color components of the images and encodes the images. In Table 5, the proposed system results are displayed on Peppers, Baboon, Lena, and Airplane color images.

Table 5: Results of proposed system based on selected criteria on color images

\begin{tabular}{|l|l|}
\hline Input Image & Entropy \\
\hline Peppers & 7.9368 \\
\hline Lena & 7.8055 \\
\hline Airplane & 7.9175 \\
\hline Baboon & 7.7632 \\
\hline
\end{tabular}


In Figures 13 and 14, histogram histograms are shown for the Lena and Baboon color images. In the form shown, the left histogram of the main images is displayed for all three color components, encoded images and decoded images. .
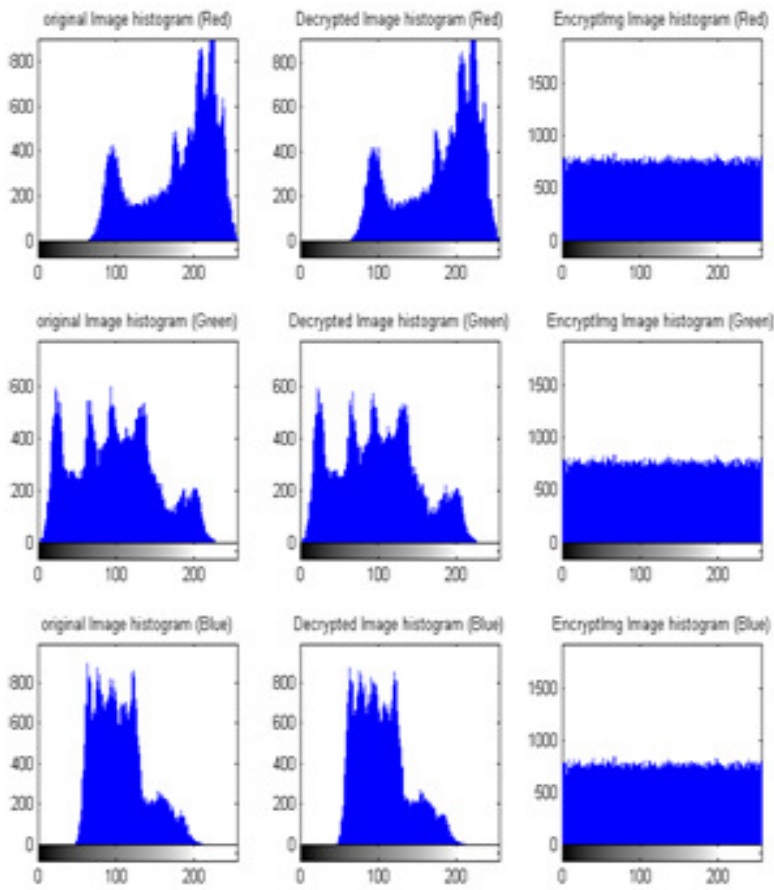

Figure 13: The histogram of the Lena color image, the left side of the main image histogram, the decoded image and the encoded image for each of the three color image components
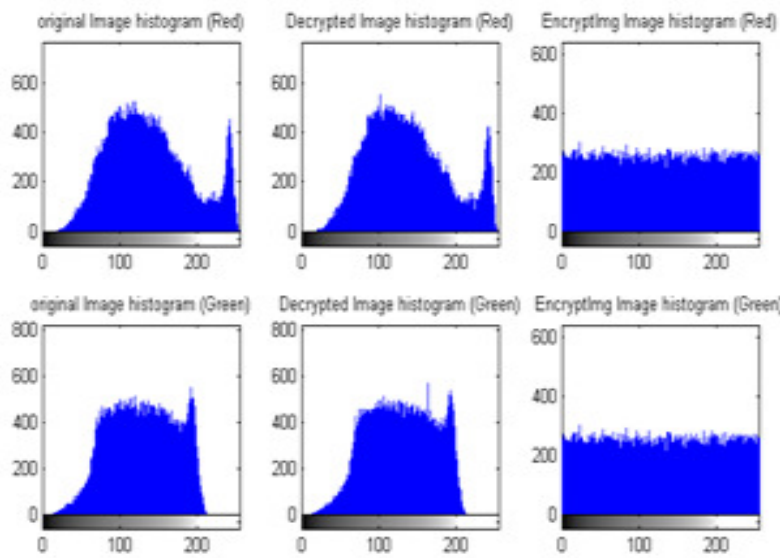

Encryptimg Image histogram (Green)
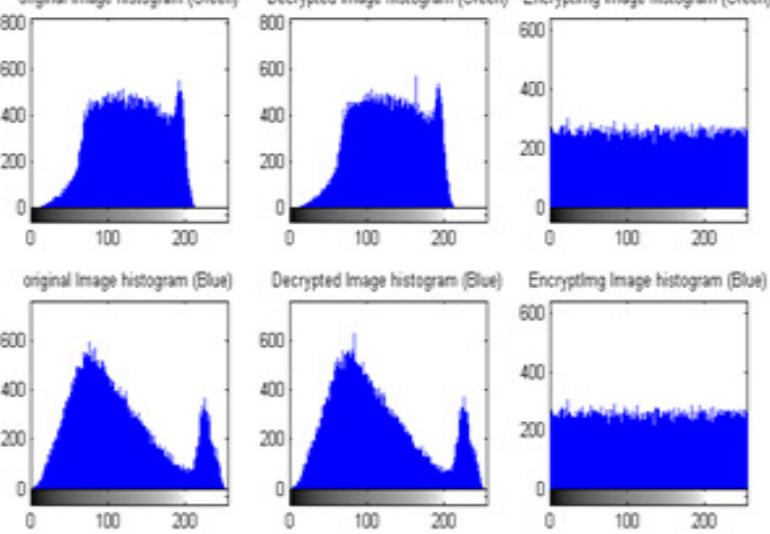

Figure 14: The Baboon color image histogram, from the left side of the main image histogram, the decoded image and the encoded image for each of the three color image components 
Table 6 shows the selected color correlations correlation coefficient.

Table 6: Results of the proposed system based on the correlation coefficient on the components of color

\begin{tabular}{|l|l|l|l|}
\hline \multicolumn{5}{|c|}{ images } \\
\hline Input Image & Red & Green & Blue \\
\hline Peppers & 0.0034 & -0.0037 & -0.0019 \\
\hline Lena & -0.0043 & -0.0056 & -0.0037 \\
\hline Airplane & -0.0022 & $-7.6437 \mathrm{e}-04$ & $4.2443 \mathrm{e}-04$ \\
\hline Baboon & 0.0032 & $-5.1269 \mathrm{e}-04$ & 0.0017
\end{tabular}

In Figure 15, several examples of encoded and decoded color images are displayed

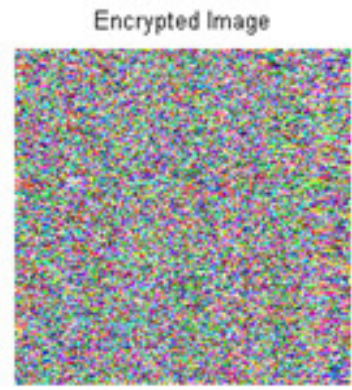

Encrypted Image

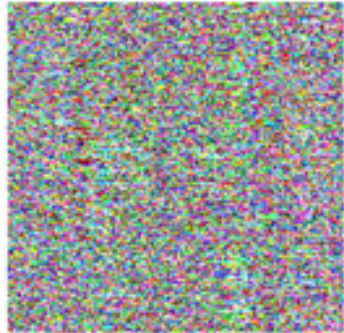

Encrypted Image

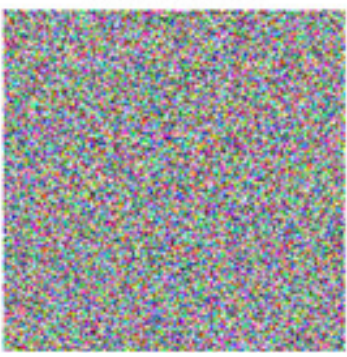

Decrypted Image

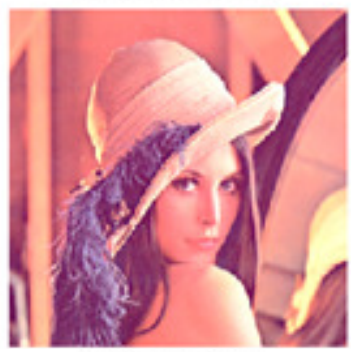

Decrypted Image

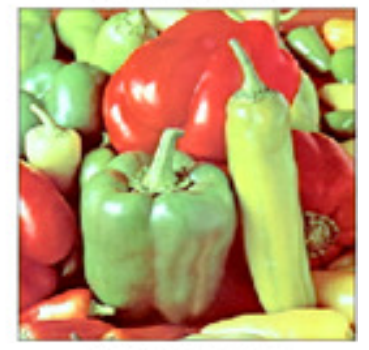

Decrypted Image

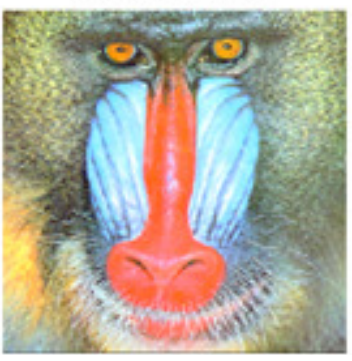

Figure 15: Output images of the proposed system on color images 


\section{CONCLUSION}

In this paper, a method was presented based on fractal theory and operators of genetic algorithm to encrypt images. At first, in the proposed method the input images are combined with Mandelbrot fractal image then, according to the size of the images, a series of random numbers are generated that in the next stage, by using these numbers in each step two rows of pixel are selected and double point combined operations are performed on them. Finally, by using random numbers generated in the second stage, the row of pixels of the image of previous step is dislocated that this act is as mutation practice in genetic algorithm and the encrypted image is obtained in this way. If input images to be colored, this act is done on all three color components. In the decode step at first, the mutation practice conducted at the encryption step is done reversely then the double point combined operation is done in reverse form. Finally, in order to achieve the original image, Mandelbrot fractal image is subtracted from the generated image. The proposed system was evaluated by using the criteria used in these areas and also was compared with similar systems and according to various criteria, the proposed system can obtain the acceptable and appropriate values based on various criteria and compared with previous similar methods, the proposed method could also get better results based on the mentioned criteria.

\section{REFERENCES}

[1] J. Weir, W. Yan, "A comprehensive study of visual cryptography", Transaction on DHMS V, LNCS, Springer, pp. 70-105, 2010.

[2] C.M. Hu, W.G.Tzeng, "Cheating prevention in visual cryptography", IEEE Transactions on Image Processing 16(1), pp. 36-45, 2007.

[3] A. Campbell, The Designer's Lexicon. Chronicle Books, San Francisco, 2000.

[4] W. Qiao, H. Yin, H. Liang, "A Kind Of Visual Cryptography Scheme For Color Images Based On Halftone Technique", International Conference on Measuring Technology and Mechatronics Automation 978-0-7695-3583-8/09, pp. 393-395, 2009.

[5] J. S. Lee, T. H. Ngan Le, "Hybrid (2, N) Visual Secret Sharing Scheme For Color Images", 978-14244-4568-4/09, IEEE, 2009

[6] P. S. Revenkar, A. Anjum, W. Z. Gandhar, "Survey of visual cryptography schemes", International Journal of Security and Its Applications, Vol, 4, No. 2, 2010.

[7] Wikipedia, "Mandelbrot Set", http://en.wikipedia.org/wiki/Mandelbrot_set.

[8] R.J. Chen, W.K. Lu, J.L. Lai, "Image encryption using progressive cellular automata substitution and SCAN", In: IEEE international symposium on circuits and systems, 2005.

[9] R. Enayatifar, "Image encryption via logistic map function and heap tree", Journal of the Physics Science, pp. 221-8, 2011.

[10] Z. Liu, L. Xu, C. Lin, J. Dai, S. Liu, "Image encryption scheme by using iterative random phase encoding in gyrator transform domains", Optics and Lasers in Engineering 49, pp.542-6, 2011.

[11] H. Li, Y. Wang, "Double-image encryption based on discrete fractional random transform and chaotic maps", Optics and Lasers in Engineering 49, pp.753-757, 2011. 
[12] Z. J. Liu, Q. Guo, L. Xu, "Double image encryption by using iterative random binary encoding in gyrator domains", Optics Express 18, pp.12033-12043, 2010.

[13] J. Koljonen, "Comparison of nearest point algorithms by genetic algorithms", Expert Systems with Applications 38, pp.10303-10311, 2011.

[14] D. Ashlock, J. Alexander Brown, "Fitness functions for thee Mandelbrot set", IEEE Transaction, 2011.

[15] http://en.wikipedia.org/wiki/Fractal.

[16] A. H. Abdullah, R. Enayatifar, and M. Lee, "A hybrid genetic algorithm and chaotic function model for image encryption," AEU - International Journal of Electronics and Communications, vol. 66, pp. $806-816,10 / / 2012$

\section{Attachments images:}
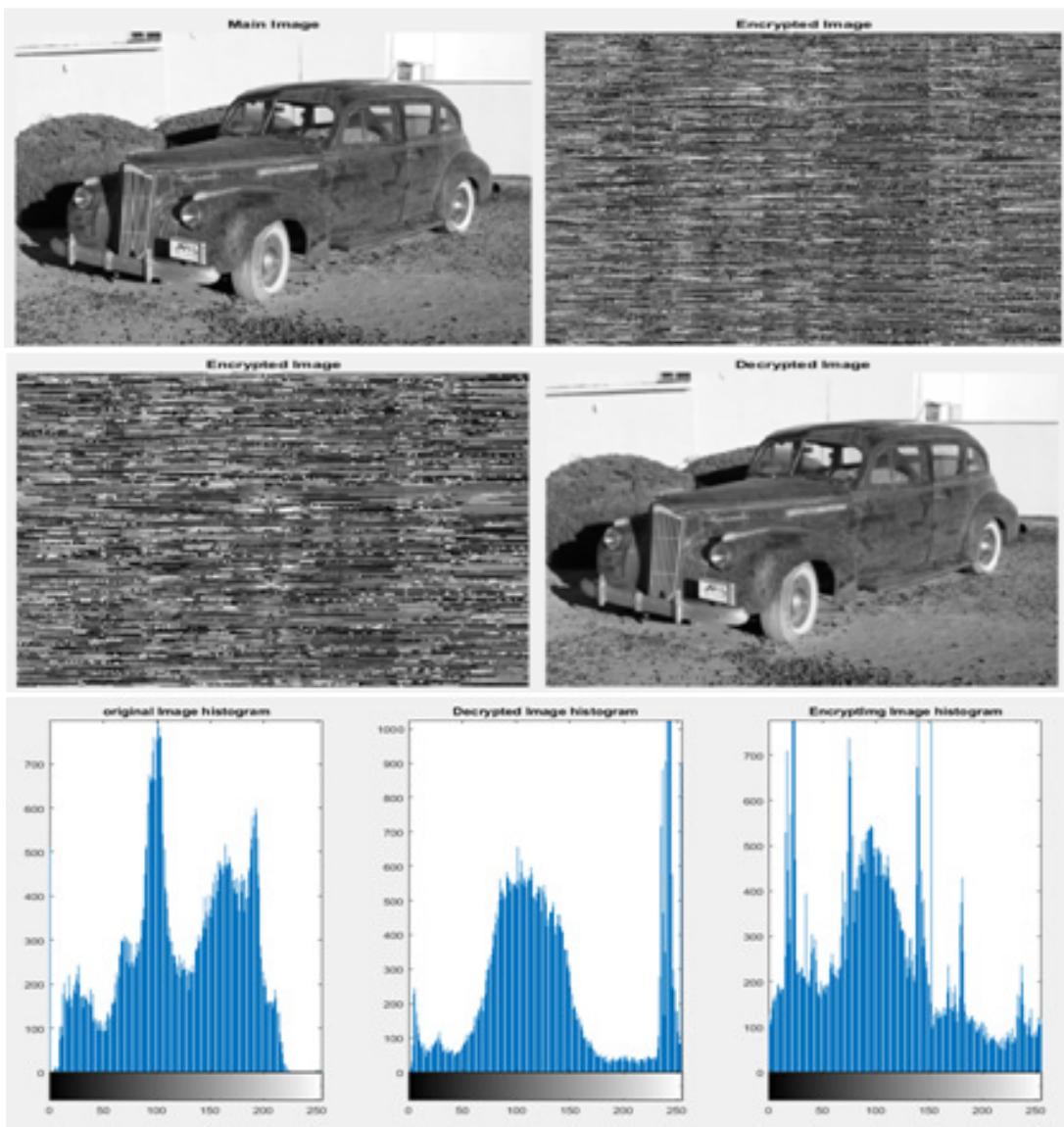
Computer Science \& Information Technology (CS \& IT)
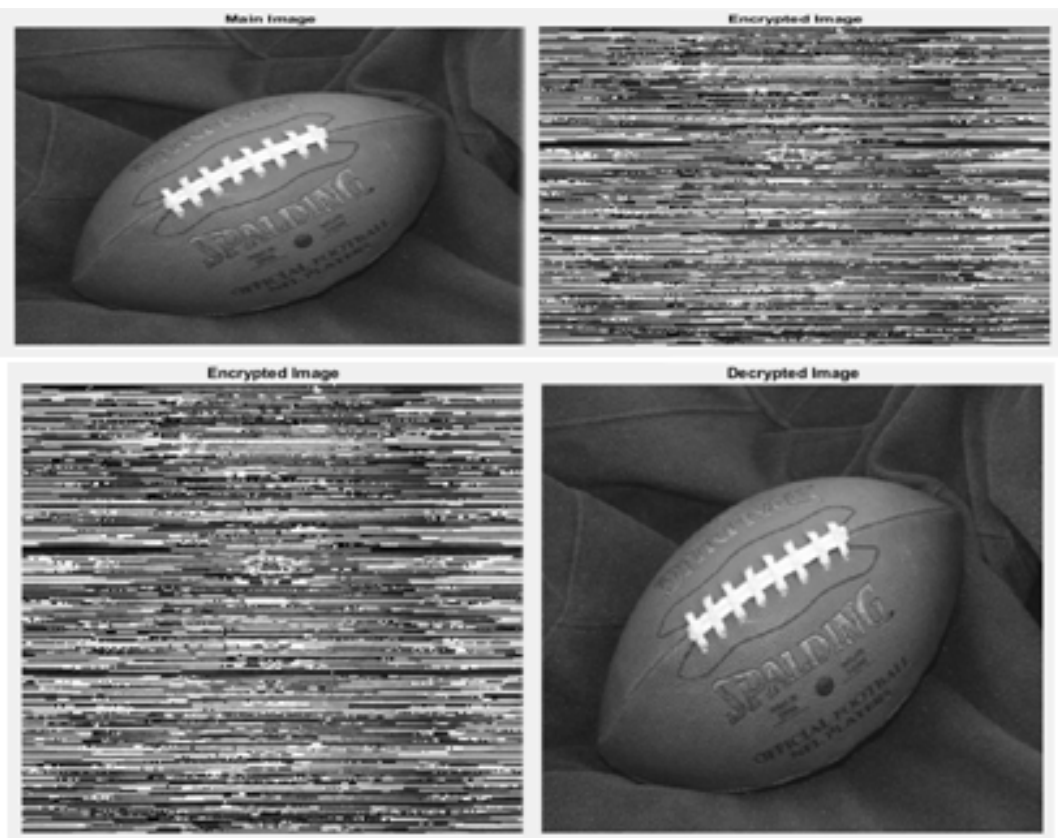